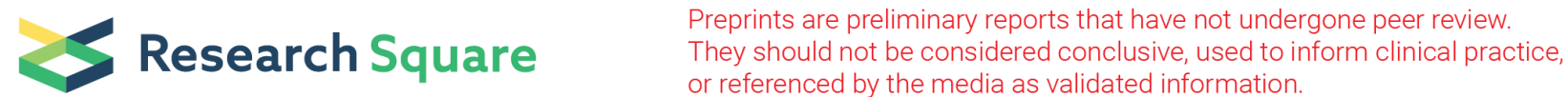

\section{Relationships between Developmental Strategies for Additional Indications and Price Revisions for Anticancer Drugs in Japan}

Hideki Maeda ( $\triangle$ maeda@my-pharm.ac.jp )

Meiji Pharmaceutical University

Research article

Keywords: anticancer drug, oncology, drug price revision, additional indications, Japan, National Health Insurance drug price

Posted Date: August 5th, 2021

DOI: https://doi.org/10.21203/rs.3.rs-779258/v1

License: (c) (1) This work is licensed under a Creative Commons Attribution 4.0 International License.

Read Full License 


\section{Abstract \\ Background}

The relationships between developmental strategies for additional indications and drug price revisions have not been thoroughly studied. Here, we investigated the price revisions for anticancer drugs approved in Japan.

\section{Methods}

The study was based on published information on anticancer drugs approved between January 2009 and March 2020 in Japan. We investigated the relationships between the pharmacological and regulatory characteristics of anticancer drugs and occurrence/non-occurrence of the Japanese National Health Insurance (NHI) price revisions.

\section{Results}

Eighty-one new anticancer drugs were given NHI price listings during the survey. On April 1, 2020, the prices of 23 anticancer drugs had been revised from the initial pricing, the prices were reduced for all, except for one drug. Several parameters showed the relationships between drug characteristics and NHI price revisions. The achievement of additional indications and compound type were identified as explanatory factors for these relationships. Additional indication profiles were defined to assess the relationships between the methods for additional indication achievement and price revisions. For the 32 drugs with additional indications, the relationships between the additional indication profiles and occurrence/non-occurrence of $\mathrm{NHI}$ price revision were investigated. The proportion of drugs with $\mathrm{NHI}$ price revisions was higher if the additional indication had more patients than if the additional indications had approximately the same numbers of patients or fewer patients.

\section{Conclusions}

$\mathrm{NHI}$ price revision was significantly related to the achievement of additional indications and compound type. The strategy for additional indications was found to affect the occurrence/non-occurrence of $\mathrm{NHI}$ price revisions.

\section{Introduction}

High drug prices are a major societal issue. In Japan, population aging is progressing more rapidly than that in other countries, and the national budget proportion associated with medical costs is increasing continuously [1]. Furthermore, Japan's healthcare system involves universal insurance coverage. Accordingly, more than $90 \%$ of approved new molecular entities are listed in the National Health 
Insurance (NHI) system and are thus eligible for $\mathrm{NHI}$ reimbursement [2]. Thus, Japan's problems with drug prices and the $\mathrm{NHI}$ are more severe than those in other countries. Moreover, the NHI pricing method in Japan is unique and highly complex. The initial pricing is, in principle, performed using one of the following methods: (i) the cost-price pricing method, involving cost price-based calculation and (ii) the similar-efficacy comparison method, involving comparison with one or more drugs with similar pharmacological activities [3]). The issue is not settled with drug price calculation, because the price is reassessed once every 2 years, often resulting in $\mathrm{NHI}$ price revision, whereby the price is usually reduced. Furthermore, from 2021, drug price reassessment will be carried out annually [4].

There are many cancer types and patient strata for which treatment is unsatisfactory, and there is a need for new anticancer drugs. Anticancer drugs are often approved for indications of orphan cancers, and their novelty often results in high pricing because of premium rewards [5]. Generally, the clinical development of an anticancer drug is for one cancer type, and often, the subsequent development strategy is to achieve additional indications after approval for the primary cancer indication $[6,7]$. Additionally, consideration is generally given to unmet medical needs and patient access to new drugs, and even if profitability is disregarded, cancer types are prioritized [7]. Therefore, in many cases, the return on the investment of anticancer drugs is expected to include not only sales for the initial indication, but also for additional indications. In the above process, drug price reduction in Japan is a hurdle to be overcome in establishing development strategies. At first glance, increasing medical costs and new drug innovation are incompatible in some areas. However, it is crucial to achieve a proper balance, and the calculation of appropriate drug prices enables the funding of research and development costs. Furthermore, it provides resources for the development of innovative drugs. The drug pricing system is important, and the aim should be to balance patient access and innovation funding [8-10]. Given this context, when establishing development strategies for anticancer drugs in Japan, including additional indications, it is crucial to consider NHI pricing methods, especially NHI price revision. However, these methods remain to be evaluated in detail. In the present study, we investigated the relationships between $\mathrm{NHI}$ price revision and pharmacological and regulatory characteristics and development strategies, represented by additional indication achievement methods, for anticancer drugs approved in Japan.

\section{Materials And Methods}

Drug selection for cancer treatment

This was a retrospective survey of anticancer drugs approved in Japan between January 2009 and March 2020. Anticancer drugs are defined as therapeutic drugs that directly target malignant tumors. Hence, drugs to treat cancer-related pain, benign tumors, or pre-cancer lesions; palliative care drugs; diagnostic drugs; drugs used before anticancer drug administration; and prophylactic drugs for adverse effects were excluded.

Data collection 
Information was collected from publicly available data. Information regarding applications for approval was mostly obtained from the Pharmaceuticals and Medical Devices Agency's website [11]. Information related to NHI prices and revisions was obtained from the Central Social Insurance Medical Council's website [12], Ministry of Health, Labour and Welfare's medical insurance website [13], NHI Drug Price Standards [14], and NHI Drug Price Standards Quick Reference Tables [15]. The pharmacological and regulatory characteristics of each anticancer drug were assessed, and an independent database was prepared. The data included the following: (i) generic name; (ii) submission-related information; (iii) administration route; (iv) therapeutic indication classification code; (v) indications; (vi) cancer type, in terms of the number of patients, as follows: major: gastric, lung, colorectal, hepatic, breast, and prostate cancer; orphan: rare diseases; and minor: neither major nor orphan; (vii) additional indication-related information; (viii) approval conditions; (ix) initial NHI price-related information; (x) sales predictions; (xi) compound type; and (xii) company. This study was conducted according to the Strengthening the Reporting of Observational Studies in Epidemiology (STROBE) reporting guidelines [16] for crosssectional studies.

Classification of methods for additional indication achievement

Methods for additional indication achievement can be classified as follows (Online Resource 1):

A: Expansion: The number of patients with an additional indication is considerably greater than that with the initial indication.

B: Retention: The number of patients with an additional indication slightly differs from that with the initial indication.

C: Targeting orphan cancers: The additional indication is an orphan cancer or the patient population for it is considerably smaller than that for the initial indication.

Occurrence/non-occurrence of $\mathrm{NHI}$ price revision

Whether $\mathrm{NHI}$ price revision occurred is defined as follows:

1. No revision: The price changes because of a consumption tax increase or is lower than $3 \%$ based on market price or similar assessments.

2. Revision occurred: The conditions in (i) do not apply and/or NHI price revisions are made based on special rules.

The $\mathrm{NHI}$ price revision is based on two major methods. One is regular price revision by a regular drug price survey, and the other is based on special rules for price revision. The special rules include premium rewards for innovative development, price reduction for long-listed drugs, market expansion-related repricing, dosage/regimen change-related repricing, repricing for indication change, and other calculations at the time of repricing for orphan drugs, pediatric indications, and genuine clinical usefulness (Online Resource 2). 
Statistical analysis were performed using Microsoft® EXCEL and JMP, with a significance level of $5 \%$. To evaluate the relationships between pharmacological characteristics and NHI price revision, Pearson's $\chi^{2}$ test was used to compare nominal variables and Student $t$-test was used to compare continuous variables. The Kaplan-Meier analysis was used for the time course of NHI price revision.

\section{Results}

Characteristics of anticancer drugs with $\mathrm{NHI}$ price listing

Totally, 153 anticancer drugs were approved in Japan between January 2009 and March 2020, including 81 new drugs and 72 with additional indications. All 81 approved new anticancer drugs had NHI price listings. The number of anticancer drugs with $\mathrm{NHI}$ price listings each year is shown, with classification by occurrence/non-occurrence of $\mathrm{NHI}$ price revision, in Fig. 1. The mean number of anticancer drugs with $\mathrm{NHI}$ price listing per year was 7.4 (range: $0-13$ ). Investigation of the occurrence/non-occurrence of $\mathrm{NHI}$ price revision for the 81 new anticancer drugs by April 1, 2020, showed revision and retention of the initial price for 23 and 58 drugs, respectively. Of the 23 drugs, the number of drugs with price revisions for different reasons, with some revisions for two or more reasons, were as follows: revision based on regular price revision, 13; market expansion-related repricing, 14; premium rewards for orphan disease indication, 4; and dosage/regimen change-related repricing: 3. For the following two drugs, the $\mathrm{NHI}$ price was increased, both of which are cases of premium rewards for orphan disease achieved as an additional indication: (i) ibrutinib: from $¥ 9,367$ to $¥ 10,135$ (increase by $8.2 \%$ ); and (ii) eribulin: from $¥ 64,070$ to $¥ 67,121$ (increase by $4.8 \%$ ). For the other 21 drugs, the NHI price was reduced; the mean reduction rate for the 23 drugs was $14.1 \%$.

Figure 1.

The pharmacological and regulatory characteristics of the 81 new anticancer drugs surveyed are shown in Table 1.

\section{Table 1. Characteristics of compounds and regulatory programs of 81 oncology drugs}

Relationships between anticancer drug characteristics and NHI price revision

The relationships between the occurrence/non-occurrence of $\mathrm{NHI}$ price revision and the pharmacological and regulatory characteristics of anticancer drugs were investigated (Table 2).

\section{- Table 2. Characteristics of drugs and NHI price revision}

Regarding the occurrence/non-occurrence of $\mathrm{NHI}$ price revision, stratified analysis was performed based on pharmacological and regulatory characteristics. Significant differences $(P<0.05)$ were observed in the presence/absence of additional indications, solid tumor versus hematologic tumor, primary indication, 
compound type, time from application for manufacturing approval until approval, and time since the initial $\mathrm{NHI}$ price listing date. In other words, anticancer drugs with $\mathrm{NHI}$ price revision were more likely to have additional indications, such as solid tumors, to have primary indications that were not orphan diseases, and, in terms of compound type, to be molecular-targeted drugs or immunotherapeutic drugs. On the contrary, anticancer drugs without $\mathrm{NHI}$ price revision were more likely to have a short time from application for manufacturing approval until approval and since the initial NHI price listing.

The Kaplan-Meier analysis was used to investigate the changes with time in the nominal variables with significant differences, as shown in Table 2. There were significant differences in additional indications and compound types (Fig. 2). The same method was used to investigate the 35 drugs for which the primary indication was an orphan cancer, and no significant differences were found (Fig. 3).]

Figure 2

Figure 3

Relationships between the type of procedure for additional indications and $\mathrm{NHI}$ price revisions The occurrence/non-occurrence of $\mathrm{NHI}$ price revision in different development strategies is shown in Table 3.

\section{Table 3. Relationship between the type of procedure for additional indications and NHI price revisions}

Type-A drugs (expansion) had more often undergone NHI price revision $(P<0.001)$. For type-A drugs, the mean revised drug price was $88.2 \%$ before revision, whereas the respective means for type-B and type- $C$ drugs were $102.8 \%$ and $99.5 \%$, respectively, indicating that there was almost no change on average for these drugs.

\section{Discussion}

$\mathrm{NHI}$ drug pricing system and price revision system

In this study, we investigated the relationships between $\mathrm{NHI}$ price revisions and anticancer drug characteristics over time. The results suggest that the achievement of additional indications and compound type are significant explanatory factors. Furthermore, although future quantitative investigations are needed, a development strategy with cancer affecting a small number of patients as the primary indication, followed by one or more major cancers as additional indications, will result in the highest drug price reduction in the Japanese drug pricing system. The NHI pricing method used in Japan is unique and complex $[1,17]$. In principle, for the initial pricing, cost price calculation and similar-efficacy comparison are made [3]. Among the drugs priced using the latter, premium rewards are added to some, such as for innovation and/or usefulness. These include orphan disease drugs and drugs for pediatric use and/or sakigake (i.e., accelerated approval of drugs designated as breakthrough therapies and that address unmet medical needs) $[18,19]$. Furthermore, revisions may include price adjustment for 
consistency with overseas prices, inter-specification adjustments, and different dosage forms [20]. This system is relatively non-transparent, because the process involves decisions made after repeated negotiations between pharmaceutical companies and governmental agencies.

The health technology assessment (HTA) performed in all developed countries has no more than a supplementary role within the Japanese drug pricing system [21]. In Japan, HTA was only introduced after a prolonged debate [22]. It was first performed at a pilot scale, and then introduced full-scale [23, 24]. Thus, it is not used when calculating drug prices; it is merely used to supplement judgment about the appropriateness of drug prices in the Japanese system. Furthermore, in the Japanese drug pricing system, the same price is not retained once it has been calculated, and the price may be reduced based on market price assessments, which are performed once every 2 years and are to be performed annually from 2021. In addition to NHI price revisions based on market prices, prices may be reassessed according to special rules, and preliminary investigations of the approach for drug price reduction have been performed $[25,26]$. These special rules are usually applied for market expansion-related repricing and price reduction for drugs listed long term. Price reduction is sometimes performed due to orphan diseases as additional indications [24], and it involves top-down decision-making, centered on the Central Social Insurance Medical Council, and without negotiations with pharmaceutical companies. Similar to the drug pricing method, the process is also non-transparent.

$\mathrm{NHI}$ price systems in other countries

Efforts to balance cost containment and oncology drug access are not unique to Japan. In 2018, China proposed a volume-based procurement program to optimize drug pricing $[27,28]$. Furthermore, the National Reimbursement Drug List was formally established in 2000 . It covers $52 \%$ of China's population under government urban health insurance programs and serves as a means of drug price negotiation for high-cost drugs [29]. Although it is potentially aimed at price renegotiation at 2-year intervals or new indication inclusion, there has been limited effect on patient access to most anticancer drugs [30]. In contrast to re-pricing in Japan, renegotiation and re-pricing of drugs occurs at either the end of the 2-year contract duration or the addition of new indications for reimbursement. Considering likely pricing pressure and market dynamics, the re-pricing often results in significant price reduction [31].

Alternatively, Korea was one of the first Asian countries to mandate pharmacoeconomic data submission for reimbursement decision-making. In Korea, reimbursement assessments and price negotiations are mandatory for new drugs. While cost effectiveness, as assessed by the Health Insurance Review of Assessment Service, is used to determine reimbursement, prices are fixed through negotiations with the National Health Insurance Service. Importantly, Korea has also implemented three methods to improve patient access to high-cost drugs: risk-sharing agreements, essential drug designation, and a waiver for cost-effectiveness analysis [32]. In the case of drug post-listing re-pricing, expanded indications also often trigger a post-listing price-cutting [33].

In several Western European countries, re-pricing is often triggered by either a new product entry or an expansion of the indications. However, in many instances, a more robust evaluation requiring an updated 
dossier and economic models is required. In these cases, the results of re-pricing are often not clear, and there is a significant variability in re-pricing although the most frequent result is price reduction [34].

The expansion of indications for an anticancer drug often resulting in re-pricing is consistent among China, Korea, and Western European countries. However, the level of detail and the focus of the evaluation process have some variabilities. Most re-pricing cases result in a price reduction and are driven by various factors such as market dynamics and the economic effect of additional volume.

Discussion on results of the present study and difficulties related to appropriate NHI price revisions In the present study on the Japanese drug pricing system, we focused on NHI price revision and factors such as the effects of drug pharmacological and regulatory characteristics, and development methods were investigated. There are several studies on factors related to premium rewards during initial drug pricing in Japan [35, 36]. However, to our knowledge, this is the first study on the factors affecting NHI price revision for anticancer drugs, methods for additional indication achievement, and NHI price revision.

Regarding development strategies for anticancer agents, development for cancer types with major unmet medical needs is invariably considered first, and such cancer types are often orphan cancers. In the Japanese drug pricing system, typically, if the indication at the time of new drug approval is an orphan cancer, the NHI drug price is awarded a premium and subsequently not readily reduced. However, the findings of the present study do not support the hypothesis that drugs for orphan cancers are not readily subject to NHI price revision (Fig. 3), suggesting that even in the case of orphan anticancer drugs, other factors lead to drug price reduction.

Future issues and proposals

In 2018, clear standards were established for additional indication-related repricing for market expansion. These standards specify that market expansion-related repricing is applicable when market expansion due to additional indications results in sales of more than $¥ 35$ billion per year. This change in the system was associated with the innovative anticancer drug nivolumab. Nivolumab, first developed in Japan, was approved for melanoma, an orphan cancer, and thus achieved a high drug price; thereafter, it was approved for non-small-cell lung cancer. Therefore, its sales increased rapidly, leading to a prompt reduction in the price by $50 \%$ as a matter of urgency [37]. We consider this price reduction to be irrational and excessive. The reasons for the significant differences based on the types of compounds, shown in Fig. 2, are that these drugs are affected by major reductions in the drug prices of similar compounds.

The changes in the drug pricing system in 2018 resulted in clear criteria for additional indication-related repricing for market expansion. However, there has been no change in the difficulty in predicting the sales associated with additional indications or the situations in which development costs can be recouped. Drug price reduction due to market expansion-related repricing has the potential to discourage innovative drug research and development [38]. 
In the United States and European Union, an indication-based pricing system has been examined recently. In this system, a drug does not have a single price, but its price is calculated separately for each indication, according to its value for that indication [39-42]. If indication-based drug pricing were to be introduced in Japan, it would probably result in a more objective and transparent system. Furthermore, when developing anticancer drugs, development starting with orphan cancers, based on unmet medical needs, is a sensible approach. From the perspective of drug pricing system or NHI price revision, it is considered unacceptable to hinder patients' access to innovative drugs. Thus, we consider indicationbased drug pricing an appropriate system.

This study had some limitations. First, this was a retrospective survey using publicly available information. Second, the study involved drugs that were approved and had NHI price listings. Drugs whose development was discontinued and had not been approved were not included. Third, the classification of development methods was qualitative, based on the number of patients and principal cancer type. In future studies, it will be necessary to perform quantitative classification with the number of patients and sales as indices.

In conclusion, we found that the presence/absence of additional indications and compound type were significant factors for the occurrence/non-occurrence of $\mathrm{NHI}$ price revision. Furthermore, the NHI price revision was influenced by the strategies for additional indication achievement for anticancer drugs. If the primary indications were rare cancers and the additional indications were cancers affecting more patients, drug prices decreased.

\section{Declarations}

Acknowledgments

We would like to thank Editage (www.editage.com) for English language editing.

Conflict of Interest

$\mathrm{HM}, \mathrm{AO}$, and MA have no conflict of interest. KS is an employee of Daiichi Sankyo.

Funding

This work was partially supported by JSPS KAKENHI (Grant Number 20K20251).

\section{References}

1. Reich MR, Shibuya K. The future of Japan's health system - sustaining good health with equity at low cost. N Engl J Med. 2015;373:1793-7.

2. Shibuya K, Hashimoto H, Ikegami N, et al. Future of Japan's system of good health at low cost with equity: beyond universal coverage. Lancet. 2011;378:1265-73. 
3. Hashimoto H, lkegami N, Shibuya $\mathrm{K}$, et al. Cost containment and quality of care in Japan: is there a trade-off? Lancet. 2011;378:1174-82.

4. Ministry of Health, Labour and Welfare. (2021) Overview of fundamental reform plan of the NHI drug price in Japan. Available at https://www.mhlw.go.jp/file/06-Seisakujouhou-12400000Hokenkyoku/0000114381_2.pdf. Accessed May 2021.

5. Gregson N, Sparrowhawk K, Mauskopf J, et al. Pricing medicines: theory and practice, challenges and opportunities. Nat Rev Drug Discov. 2005;4:121-30.

6. Maeda $\mathrm{H}$, Kurokawa T. Acceptance of surrogate end points in clinical trials supporting approval of drugs for cancer treatment by the Japanese regulatory agency. Ann Oncol. 2015;26:211-6.

7. Shibata S, Noguchi E, Matsushita M, et al. Can rare cancer drugs expect sales in Japan?: a prescription pattern analysis of drugs for chronic myelogenous leukemia and neuroendocrine tumor. Journal of Regulatory Science. 2019;7:1-9.

8. Schoonveld $\mathrm{E}$. The price of global health: drug pricing strategies to balance patient access and the funding of innovation. New York, pp: Taylor \& Francis; 2016.

9. Suleman F, Low M, Moon S, et al. New business models for research and development with affordability requirements are needed to achieve fair pricing of medicines. BMJ. 2020;368:14408.

10. Grignolo A, Mingping Z. Pharma opportunities and risks multiply as regulatory reform remakes APAC: Expanded accelerated pathways challenge developer value story, evidence collection, and market access strategies. Ther Innov Regul Sci. 2018;52:514-22.

11. Pharmaceuticals and Medical Devices Agency. (2004) Prescribed drug information search. Available at https://www.pmda.go.jp/PmdaSearch/iyakuSearch/ (in Japanese). Accessed May 2021.

12. Ministry of Health, Labour and Welfare. (2021) Central council of social insurance medical services. Available at https://www.mhlw.go.jp/stf/shingi/shingi-chuo_128153.html (in Japanese). Accessed May 2021.

13. Ministry of Health, Labour and Welfare. (2021) Medical care insurance. Available at https://www.mhlw.go.jp/stf/seisakunitsuite/bunya/kenkou_iryou/iryouhoken/index.html (in Japanese). Accessed on May 2021.

14. Yakuji Nipposha. NHI Drug Price Standards version October 2019. Yakuji Nippo, Ltd.; 2019. (in Japanese).

15. Jiho. (2020) NHI Drug Price Standards quick reference tables. Jiho Inc. (in Japanese).

16. Vandenbroucke JP, von Elm E, Altman DG, et al. Strengthening the reporting of observational studies in epidemiology (STROBE): explanation and elaboration. Epidemiology. 2007;18:805-35.

17. Takayama A, Narukawa M. Pharmaceutical pricing and reimbursement in Japan: For faster, more complete access to new drugs. Ther Innov Regul Sci. 2016;50:361-7.

18. Kajiwara E, Shikano M. Considerations and regulatory challenges for innovative medicines in expedited approval programs: breakthrough therapy and Sakigake designation. Ther Innov Regul Sci. 2020;54:814-20. 
19. Tanaka $M$, Idei $M$, Sakaguchi $H$, et al. Achievements and challenges of the Sakigake designation system in Japan. Br J Clin Pharmacol (in press). 2021. doi:10.1111/bcp.14807.

20. Takayama A, Kobayashi E, Nakamura T, et al. Quantitative assessment of premium rates for clinical usefulness in new drug price calculation in Japan. Ther Innov Regul Sci. 2017;51:582-8.

21. Takura T. An evaluation of clinical economics and cases of cost-effectiveness. Intern Med. 2018;57:1191-200.

22. Shiroiwa T, Fukuda T, Ikeda S, et al. New decision-making processes for the pricing of health technologies in Japan: the FY 2016/2017 pilot phase for the introduction of economic evaluations. Health Policy. 2017;121:836-41.

23. Kido K, Matsumaru N, Tsukamoto K. Health technology assessment in Japan: a pharmaceutical industry perspective. Ther Innov Regul Sci. 2019;53:472-80.

24. Hasegawa M, Komoto S, Shiroiwa T, et al. Formal implementation of cost-effectiveness evaluations in Japan: A unique health technology assessment system. Value Health. 2020;23:43-51.

25. Fukumoto D, Tsuyuki A, Suzuki T. Drugs targeted for price cutting in Japan: the case of price revisions based on the divergence of official versus delivery prices. Ther Innov Regul Sci. 2017;51:597-603.

26. Shibata S, Fukumoto D, Suzuki T, et al. Analyzing upward deviation of actual vs predicted drug sales in Japan for a reasonable drug-pricing policy. Ther Innov Regul Sci. 2020;54:544-51.

27. Hasan SS, Kow CS, Dawoud D, et al. Pharmaceutical policy reforms to regulate drug prices in the Asia Pacific region: The case of Australia, China, India, Malaysia, New Zealand, and South Korea. Value Health Reg Issues. 2019;18:18-23.

28. Tang M, Song P, He J. Progress on drug pricing negotiations in China. Biosci Trends. 2020;13:464-8.

29. Li H, Liu GG, Wu J, et al. Recent pricing negotiations on innovative medicines pilot in China: Experiences, implications, and suggestions. Value Health Reg Issues. 2018;15:133-7.

30. Guan $X$, Zhang $Y$, Wushouer $H$, et al. Differences in reimbursement listing of anticancer therapies in China: an observational study. BMJ Open. 2020;10:e031203.

31. Tang M, Song P, He J. Progress on drug pricing negotiations in China. Biosci Trends. 2020;13:464-8.

32. Kim S, Lee JH. Price-cutting trends in new drugs after listing in South Korea: The effect of the reimbursement review pathway on price reduction. Healthcare (Basel). 2020;8:233.

33. Nayroles G, Frybourg S, Gabriel S, et al. Unlocking the potential of established products: toward new incentives rewarding innovation in Europe. J Mark Access Health Policy. 2017;5:1298190.

34. Yoo SL, Kim DJ, Lee SM, et al. Improving patient access to new drugs in South Korea: Evaluation of the national drug formulary system. Int J Environ Res Public Health. 2019;16:288.

35. Shibata S, Uemura R, Suzuki T. Impact of premium rewards for the promotion of innovative drug discovery on the Japanese pharmaceutical market: An analysis by therapeutic area. Ther Innov Regul Sci. 2016;50:49-55. 
36. Shibata S, Uemura R, Suzuki T. Factors that affect the acquisition of reward premiums for promotion of innovative drug discovery in Japan. Ther Innov Regul Sci. 2016;50:56-65.

37. Fukuda A, Igarashi A. Universal health coverage and cancer drugs - A cost-effectiveness perspective (in Japanese). Gan To Kagaku Ryoho. 2016;43:1311-5.

38. Shibata S, Uemura R, Suzuki T. Evaluating the effectiveness of repricing for market expansion in the Japanese drug pricing system. Ther Innov Regul Sci. 2016;50:751-8.

39. Chandra A, Garthwaite C. The economics of indication-based drug pricing. N Engl J Med. 2017;377:103-6.

40. Pearson SD, Dreitlein WB, Henshall C, et al. Indication-specific pricing of pharmaceuticals in the US healthcare system. J Comp Eff Res. 2017;6:397-404.

41. Flume M, Bardou M, Capri S, et al. (2016) Feasibility and attractiveness of indication value-based pricing in key EU countries. J Mark Access Health Policy 4.

42. Campillo-Artero C, Puig-Junoy J, Segú-Tolsa JL, et al. Price models for multi-indication drugs: a systematic review. Appl Health Econ Health Policy. 2020;18:47-56.

\section{Tables}

[Please see the supplementary files section to view the tables.]

\section{Figures}

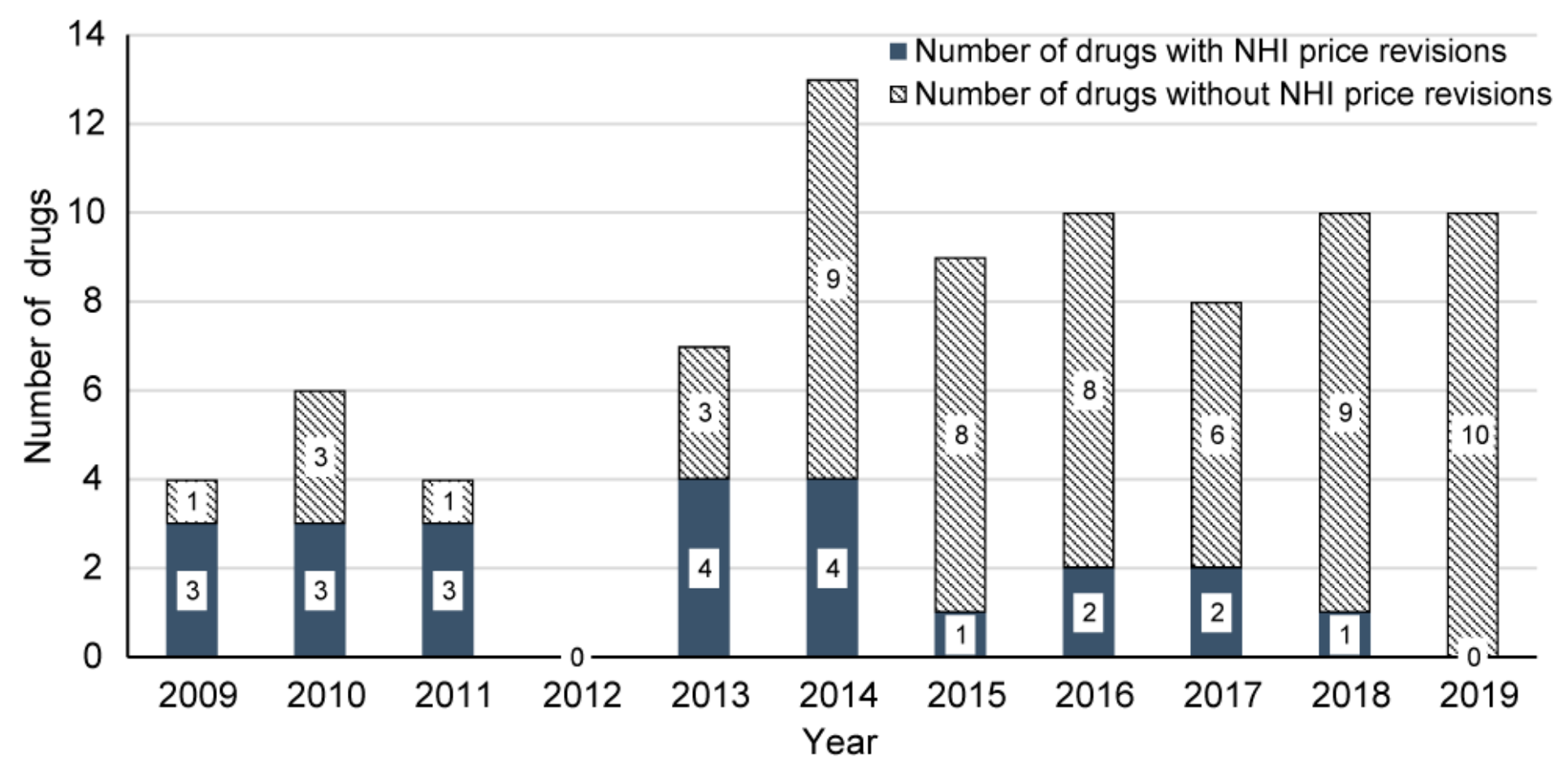

Figure 1 
Number of oncology drugs in the National Health Insurance price list and price revisions by year


Figure 2

Kaplan-Meier curve for National Health Insurance price revisions by stratification of drug characteristics. Left panel: Stratified by additional indication, $\mathrm{P}<0.001$, log-rank test, Median time for an additional indication: 2410 days Right panel: Stratified by compound type $P<0.001$, log-rank test, Median time for hormonal agent: 3050 days, Median time for cytotoxic drug: 3484 days, Median time for molecularly targeted drug: 3638 days, Median time for immunotherapy drug: 1141 days

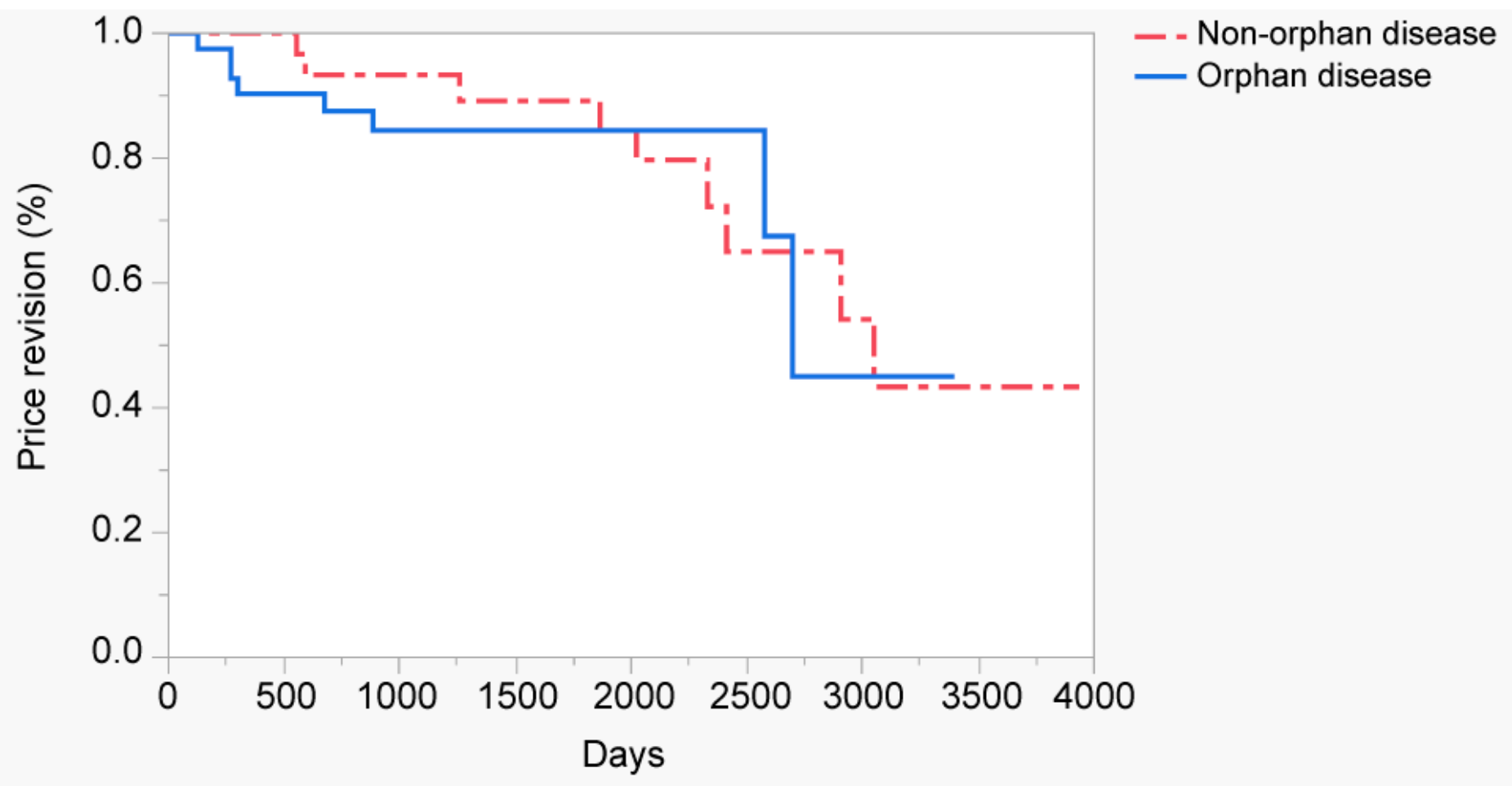

Figure 3

Kaplan-Meier curve for National Health Insurance price revisions by stratification of drug characteristics. Stratified by first indication: Orphan or non-orphan cancer $P=0.7682$, log-rank test, Median time for an orphan cancer: 2695 days, Median time for a non-orphan cancer: 3051 days

\section{Supplementary Files}


This is a list of supplementary files associated with this preprint. Click to download.

- Table1.xlsx

- Table2.xlsx

- Table3.xlsx

- ESM1.docx

- ESM2.docx 